\title{
Las rentas mínimas de inserción en época de crisis. ¿Existen diferencias en la respuesta de las comunidades autónomas?
}

\author{
Noemi Bergantiños \\ Universidad del País Vasco / Euskal Herriko Unibertsitatea. Departamento de Sociología \\ y Trabajo Social \\ noemi.bergantinos@ehu.eus

\section{Raquel Font \\ Amaia Bacigalupe} \\ Universidad del País Vasco / Euskal Herriko Unibertsitatea. Departamento de Sociología II \\ raquel.font@ehu.eus; amaia.bacigalupe@ehu.eus
}

\section{Resumen}

En el presente artículo, realizamos un análisis de la reciente evolución de las rentas mínimas de inserción (RMI) en el conjunto de las comunidades autónomas de España. El trabajo ofrece una clasificación de estas según las características de su política de RMI, tanto en el inicio de la crisis (2008) como respecto a su evolución hasta el año 2014. Se han analizado variables relativas a las dimensiones de recursos y a las coberturas, a los rasgos básicos y a las condiciones de acceso, con el fin de conocer las características, el desarrollo reciente y la capacidad de respuesta de esos programas respecto a la crisis. Los resultados muestran que, si bien los programas de RMI en su conjunto experimentan una cierta mejoría, persisten grandes diferencias autonómicas, ciertas desigualdades sociales en el acceso a las mismas y un impacto aún limitado como herramienta para paliar la pobreza y la exclusión social.

Palabras clave: rentas mínimas; crisis; desigualdades sociales; protección social; estado de bienestar

* Este trabajo ha sido financiado parcialmente por el Ministerio de Economía y Competitividad. Ayudas Programa Estatal de I+D+i Orientada a los Retos de la Sociedad (CSO201344886-R). 
Abstract. Minimum Insertion Income Programmes in Times of Crisis: Are There Any Differences Among Autonomous Communities?

This article examines the recent evolution of the minimum insertion income (MII) programmes in all of Spain's autonomous communities. The autonomous communities are classified according to the specifications of their MII programme policies at the beginning of the crisis (2008) and their evolution until 2014. For this purpose, variables related to resources, coverage, basic features and access requirements are analysed to determine the characteristics, recent development and ability of these programmes to respond to the economic crisis. The results reveal that although MII programmes as a whole show some improvement, major differences remain between autonomous communities, as well as some social inequalities regarding access to the programmes, thus suggesting that they have a limited impact as a tool to reduce poverty and social exclusion.

Keywords: minimum incomes; crisis; social inequalities; social welfare; welfare state

\begin{aligned} & \multicolumn{2}{c}{ Sumario } \\ & 1. Introducción 4. Metodología \\ & 2. Contexto de crisis y respuestas basadas 5. Resultados \\ & en la austeridad 6. Conclusiones y discusión \\ & 3. Las RMI como mecanismo Referencias bibliográficas \\ & de protección social. Aproximación \end{aligned}
a la realidad autonómica española

\section{Introducción}

En los últimos años, ha crecido de manera considerable el interés por conocer el impacto de la crisis en las diferentes esferas de la realidad social. Más exactamente, algunos trabajos se han centrado en analizar el peso y la función de las políticas públicas para corregir los efectos de dicha crisis. Buena parte de ellos apuntan hacia, por un lado, la importancia de la actuación y la respuesta de las instituciones públicas y, por otro lado, hacia la existencia de ciertas diferencias en las respuestas de las mismas y sus consecuencias (Bacigalupe et al., 2015a). En este sentido, no son pocos los trabajos que tratan de analizar con perspectiva territorial los resultados de las diferentes políticas públicas implementadas en los últimos años (Díaz-Pulido et al., 2012).

En el contexto del Estado español, han sido varios los estudios que han analizado las diferencias territoriales respecto a la respuesta a la crisis. En concreto, en un estudio anterior (Bacigalupe et al., 2015b), sometimos a estudio las diferencias entre comunidades autónomas a partir de las políticas públicas implementadas en el ámbito sanitario en el actual contexto de crisis, y los resultados obtenidos muestran como algunas comunidades autónomas (La Rioja o Madrid, entre otras) promueven políticas de austeridad y privatización sanitaria de una manera más intensa que otras. Estudios similares realizados 
con relación a, por ejemplo, las políticas de igualdad (Paleo y Alonso, 2014), el ámbito de la educación o la vivienda (Méndez et al., 2015) apuntan también a este comportamiento autonómico diferente en la implementación de políticas públicas en contextos de crisis.

En el presente artículo, queremos ahondar en esta línea de trabajo realizando un análisis de los programas de rentas mínimas de inserción (RMI) como una de las actuaciones principales en contextos de vulnerabilidad económi$\mathrm{ca}$, deterioro de las condiciones de vida y descenso de las rentas en la actual coyuntura de crisis económica (CES, 2016). Las RMI constituyen la última red de protección de los estados del bienestar, y su objetivo principal consiste en cubrir las necesidades básicas para la supervivencia de la población (Sanzo, 2013). Más exactamente, los dispositivos de rentas mínimas contemplan una prestación económica básica para aquellas personas y para aquellos hogares que presentan una insuficiencia de recursos y, por tanto, una situación de pobreza (Arriba González de Durana, 2014). Hemos optado por centrarnos en ese programa dado su importante desarrollo como mecanismo de lucha contra la pobreza y la exclusión social (EAPN, 2010), dimensiones especialmente vulnerables a los efectos de las crisis (Fernández, 2015). Concretamente, el objetivo del presente trabajo es describir la evolución que se ha producido en estos programas de rentas mínimas de inserción (RMI) durante el periodo de crisis económica (2008-2014), destacando las diferencias entre las comunidades autónomas de España. De esta manera, tratamos de contribuir a profundizar en trabajos similares, como el realizado por Fernández Maíllo (2013), aportando más indicadores de análisis, la actualización de datos y aumento del periodo de análisis y la construcción de una clasificación de las comunidades autónomas según la intensidad de su política de RMI, tanto en el inicio de la crisis como en su evolución hasta la actualidad.

\section{Contexto de crisis y respuestas basadas en la austeridad}

Explorar la naturaleza de la actual crisis requiere abandonar un enfoque exclusivamente centrado en la liberalización y en la quiebra del sistema financiero para advertir sobre su naturaleza multidimensional, global y sistémica (Pérez Orozco, 2012), así como sobre su impacto no solo económico-financiero, sino también político, social y medioambiental (Bacigalupe et al., 2015a; Méndez et al., 2015; Recio, 2013; Márquez, 2012; Subirats, 2012). A diferencia de las respuestas políticas ofrecidas en contextos de crisis en el pasado, que se basaron fundamentalmente en la expansión y el crecimiento como medidas principales para garantizar la cohesión y la protección social (Pontusson y Raess, 2002; Bieling, 2012; Shahidi, 2015), desde su inicio, la respuesta a la actual crisis se ha caracterizado por la aplicación de las conocidas como politicas de austeridad, asentadas bajo los principios de un neoliberalismo tardío (Rolnick, 2013) y basadas en un conjunto de medidas que apuestan por reducir el endeudamiento de los estados a partir de una menor inversión en el gasto público. 
La implementación de estas medidas y su impacto social también ha sido objeto de estudio en los últimos años, bajo la perspectiva de la tensión originada entre las políticas de austeridad y los principios de un estado social (Gordillo, 2013). Estos trabajos tienden a señalar que, junto con los recientes procesos de privatización de servicios públicos y recortes en el gasto de las administraciones (Díaz-Chao et al., 2015), las crecientes tasas de desempleo, el aumento de la pobreza y de las desigualdades sociales (Intermón-Oxfam, 2016; Arriba González de Durana, 2014), especialmente en el caso de los países del sur de Europa, ponen en cuestión la eficacia de las actuales políticas de austeridad impulsadas en la UE como solución a la crisis (Flores y Nieto, 2013).

En el caso español, es a partir de 2006 cuando comienzan a evidenciarse los primeros síntomas de la crisis, siendo el año 2008 el que determina su entrada en un proceso de recesión económica. A partir de esta fecha, algunos indicadores recogidos por el Instituto Nacional de Estadística muestran la dureza de los efectos de dicha crisis sobre la población española, que alcanzó tasas de desempleo muy elevadas (un $23,60 \%$ en hombres y un $25,43 \%$ en mujeres en 2014 , frente a un $10,05 \%$ en hombres y un $12,84 \%$ en mujeres registrado en 2008), también para las personas jóvenes (un 50,97\% en hombres y un $49,58 \%$ en mujeres en 2014), así como un relevante aumento de la tasa de pobreza especialmente infantil, que, en 2012 , alcanza ya un $28,3 \%$ de los niños y un $25 \%$ de las niñas.

Políticamente, las medidas de austeridad comienzan a implementarse en el Estado español a partir de 2010, tras el anuncio de recortes en las pensiones, en el área desarrollada por la Ley de dependencia o con medidas tales como la supresión del conocido como cheque bebé, entre otros. Estas medidas han continuado posteriormente, ya de la mano del Partido Popular — en el Gobierno desde 2011-, acompañadas, desde 2012, del rescate al sector financiero español dirigido desde Bruselas por un valor de 100.000 millones de euros y la reforma del sistema laboral, lo cual ha incrementado de manera importante la ya presente precariedad y el paro laboral de la población española.

\section{Las RMI como mecanismo de protección social. Aproximación a la realidad autonómica española}

En el contexto europeo, la renta mínima es considerada una herramienta fundamental para promover la inclusión e impulsar la lucha contra la pobreza y la exclusión social. El propio Parlamento Europeo es quien anima a los Estados miembros a implementar el derecho a un sistema de renta mínima entendida como una estrategia dirigida no solo a aliviar situaciones de precariedad, sino también a prevenir escenarios de pobreza y fragilidad social en el futuro (Parlamento Europeo, 2009; Comisión Europea, 2014). En la actualidad, 24 de los 27 Estados miembros cuentan con un sistema propio de renta mínima, si bien se aprecian variaciones importantes en aspectos tales como la cobertura, las cuantías o la propia disposición institucional (Deeming, 2015). 
En el caso del Estado español, las rentas mínimas autonómicas tienen su origen a finales de la década de 1980 y principios de la de 1990, con el objetivo de instituirse como última red de seguridad para la población (Aguilar et al., 1995; EAPN, 2010). La Comunidad Autónoma del País Vasco fue la pionera en implementar esta política, cuya cobertura actual resulta mucho mayor a la ofrecida en otras comunidades del Estado (Moreno et al., 2015). Así, en la mayoría de los casos, la realidad de estos programas se aleja de los estándares definidos a nivel europeo respecto a criterios tales como su definición como derecho, la cobertura o la ilimitación temporal, y su desarrollo muestra como características principales una importante variabilidad y heterogeneidad territorial (Laparra, 2013; Lavía et al., 2014; Fernández Maíllo, 2013), lo que dificulta una lectura global de la realidad de las rentas mínimas en España.

El análisis de los elementos que constituyen la actual política de RMI nos permite hacer referencia a, en primer lugar, la responsabilidad de cada comunidad autónoma en la definición y provisión de este dispositivo (Figari et al., 2013), de manera que cada una dispone de un modelo propio de renta mínima con un planteamiento normativo, una implementación y un desarrollo particulares. En segundo lugar, cabe destacar las diferencias en cuanto a la generosidad de las cuantías definidas en las RMI y, como consecuencia, el alcance de las mismas respecto a la población potencialmente beneficiaria (Fuenmayor y Granell, 2013). Por otro lado, desde el comienzo de la crisis, se observa que, mientras el incremento del desempleo se acompaña de una mayor demanda de esta prestación de mínimos, varias comunidades autónomas han introducido reformas legislativas y cambios respecto a las condiciones de acceso y a la cuantía o duración de la misma que permite distinguir entre aquellas que tienden a fortalecer el estatus de su renta mínima y las que tan solo buscan ajustar sus programas al incremento de la demanda (Arriba González de Durana, 2014). En este sentido, el reconocido aumento del número de prestaciones en el contexto de crisis no ha resultado suficiente para aliviar las situaciones causadas por el también creciente desempleo (Ayala, 2012). Finalmente, la atención sobre el número de beneficiarios de la RMI también muestra que las personas titulares de la prestación han ido en aumento en los últimos años, así como el gasto total ejecutado, si bien no todas las comunidades autónomas incrementan su gasto anual por persona beneficiaria (CES, 2016).

Al comienzo de la crisis, algunos estudios ya alertaban sobre la necesidad de actuar en mecanismos tales como las RMI (Arriba y Guinea, 2008 Laparra, 2013). Advertían sobre sus deficiencias y lagunas respecto a la protección de algunos grupos de población (Ayala, 2012), así como sobre su escasa capacidad de respuesta en referencia al aumento de las necesidades económicas de una parte de la población (Laparra y Ayala, 2009) para corregir las, después reconocidas, desigualdades sociales generadas y agudizadas por la crisis. Más adelante, otros trabajos constataban que la actuación realizada durante la crisis, lejos de mejorar la situación, había aumentado la fragilidad del sistema de RMI como herramienta de protección contra la pobreza y la exclusión social (Fernández Maíllo, 2013). En concreto, se señala que la RMI no tiene apenas 
incidencia sobre los colectivos situados ahora en el eje de exclusión, aquellos que protagonizan formas de nueva pobreza, precarización y el conocido como working poor, que acompaña y agudiza el propio contexto de crisis actual (Gutiérrez, 2014). Más aún, se apunta a que las mismas están desarrollando cada vez más una función orientada a suplir la insuficiencia de las rentas obtenidas por el trabajo por los cada vez más frecuentes colectivos inmersos en el mercado de trabajo, pero de manera precaria (Zalakain, 2014). Aquí, algunos trabajos tratan de señalar la importancia de las políticas de inclusión que acompañan prestaciones tales como la renta mínima de inserción y la creciente incidencia del recorte en gasto social, que, junto a las medidas de flexibilización del mercado de trabajo y el aumento de la precariedad, dificulta los objetivos de las políticas y de los procesos de inclusión social y de lucha contra la pobreza en los que se ubican estas RMI (De la Cal y De la Fuente, 2010).

Al hilo de estos estudios, el trabajo que presentamos aquí trata de profundizar en la discusión en torno a la actuación sobre las RMI como herramientas de lucha contra la pobreza y la exclusión. Para ello, en el siguiente apartado, se muestra el análisis realizado con el objetivo principal de clasificar las comunidades autónomas del Estado español en función de la intensidad de su política de RMI (baja, media e intensa), tanto en el comienzo de la crisis como en relación con el cambio acontecido durante el periodo de crisis.

\section{Metodología}

\subsection{Diseño de la investigación y fuentes de datos}

En una primera fase, se realizó una búsqueda y un análisis de indicadores cuantitativos y cualitativos acerca de la evolución de las RMI durante el periodo de crisis económica (2008-2014) por comunidades autónomas, a partir, fundamentalmente, de los informes de rentas mínimas de inserción del Ministerio de Sanidad, de los Servicios Sociales e Igualdad, publicados por el Ministerio de Sanidad y Política Social. Para el cálculo de algunos indicadores, se utilizó también información padronal de los años 2008-2014 y la Encuesta de Condiciones de Vida (ECV) de los años 2009-2014 del Instituto Nacional de Estadística. Además, se utilizaron informes o artículos que ya habían sido publicados anteriormente y que comparaban la situación entre comunidades autónomas para identificar posibles indicadores de interés y comprobar el grado de coincidencia con los resultados obtenidos en la presente investigación. En una segunda fase, se consultaron las páginas web y se contactó telefónicamente con algunas comunidades autónomas para ampliar y contrastar la información.

\subsection{Variables}

Las variables se agruparon en tres dimensiones principales, que fueron las que caracterizaron a las RMI: 


\section{Recursos y cobertura de la RMI}

- Gasto anual por habitante en RMI: gasto ejecutado cada año en RMI en relación con el número total de habitantes en cada comunidad autónoma, corregido por el cambio en el índice de precios al consumo (IPC) a lo largo del periodo. En el caso del País Vasco, se incluye la prestación complementaria de vivienda (PCV).

- Tasa de cobertura sobre población en riesgo de pobreza: número total de personas perceptoras (titulares y beneficiarias) de la prestación por cada cien personas en riesgo de pobreza según la Encuesta de Condiciones de Vida. La falta de disponibilidad de datos para la población en riesgo de pobreza con anterioridad a 2009 restringió su cálculo al periodo 2009-2014. Asimismo, la falta de información sobre el número de personas beneficiarias para La Rioja obligó a aplicar el promedio de la relación entre titulares y número de personas beneficiarias del conjunto de comunidades autónomas a dicho ente territorial.

\section{Características básicas de la RMI}

- Cuantía básica de la prestación, corregida por el cambio en el índice de precios al consumo (IPC) a lo largo del periodo.

- Duración de la prestación: periodo de tiempo a lo largo del cual se puede percibir la prestación.

- Garantía de financiación, distinguiendo entre prestación garantizada (prestación del servicio público de servicios sociales exigible como derecho subjetivo) y prestación no garantizada (prestación del servicio público de servicios sociales, que únicamente será exigible en el caso de que exista disponibilidad presupuestaria). Hasta 2010, el término utilizado en los informes que publicaba el Ministerio no era prestación garantizada, sino derecho subjetivo.

\section{Condiciones de acceso a la RMI}

A partir de la propuesta de Fernández Maíllo (2013), se distinguieron los conceptos siguientes:

- Persona perceptora: identifica al titular de la prestación y distingue a individuos de unidades de convivencia familiares.

- Edad mínima a partir de la cual se puede solicitar la prestación.

- Prueba de rentas, en la que se tuvieron en cuenta los aspectos siguientes:

- El porcentaje de referencia en relación con el indicador público de renta de efectos múltiples (IPREM) o con el salario mínimo interprofesional (SMI) para el cálculo de la RMI.

- La congelación de las cuantías.

- El establecimiento de topes máximos.

- La incorporación de ingresos de miembros de la unidad de convivencia para el cálculo. 
- El establecimiento de cantidades de referencia.

- El aumento del mínimo de la cuantía básica.

- Tiempo de cohabitación de la unidad de convivencia o familiar.

- Tiempo de empadronamiento en la comunidad autónoma donde se solicita la prestación.

- Tiempo de residencia efectiva en la comunidad autónoma donde se solicita la prestación.

\subsection{Análisis}

Teniendo en cuenta la probable heterogeneidad entre comunidades autónomas en relación con la intensidad de las RMI, tanto al comienzo del periodo como en su evolución posterior durante la crisis económica, se consideró relevante considerar ambos aspectos para construir la tipología final de comunidad autónoma.

\section{Identificación de la intensidad de la RMI en el comienzo del periodo}

Se tuvieron en cuenta los indicadores cuantitativos disponibles que permitían estimar la intensidad de la RMI en 2008: el gasto por habitante, la tasa de cobertura con relación a la población en riesgo de pobreza y la cuantía básica de la prestación. Asimismo, se tuvo en cuenta si la financiación de la prestación estaba o no garantizada.

En base a los resultados de cada indicador en 2008 (y en 2009, en el caso de la cobertura a población en riesgo de pobreza), se valoró a cada comunidad autónoma con una puntuación de 1 a 3 , en función de la intensidad del mismo a partir del $20 \%$ de la media del conjunto de la distribución en tal indicador. De esta forma, se evaluó con un 1 a las comunidades autónomas que alcanzaron un valor del $80 \%$ de la media, con un 2 a aquellas que se situaron entre el 80 y el $120 \%$ de la media y con un 3 a aquellas que se situaron por encima del $120 \%$ de la media del conjunto. Una vez puntuadas todas las dimensiones, se realizó un promedio que dio lugar a la siguiente escala de intensidad de RMI al comienzo del periodo:

- Comunidades autónomas con una política débil de RMI, es decir, con un promedio de 1 a 1,5 .

- Comunidades autónomas con una política media de RMI, es decir, con un promedio de 1,6 a 2,5.

- Comunidades autónomas con una política intensa de RMI, es decir, con un promedio de 2,6 a 3 .

\section{Identificación del cambio durante el periodo de crisis}

Se calculó el cambio en cada una de las tres dimensiones, así como el cambio general. Para valorar la intensidad del cambio, se asignó un valor a cada indicador $(-2,-1,0,1$ o 2$)$, según su mejora o su empeoramiento a lo largo del periodo. La asignación se modificó en función del tipo de variable: 
- En el caso de las variables cuantitativas, la puntuación del cambio de cada comunidad autónoma en cada indicador vino determinada por un valor que el equipo de investigación eligió a priori como indicativo de un cambio relevante: en el gasto por habitante y la tasa de cobertura, se estableció un aumento o una disminución del $50 \%$ para clasificar a las comunidades autónomas como $1 \mathrm{o}-1$, y un aumento o una disminución del 100\% como 2 o -2. En el caso del cambio de la cuantía básica, se estableció una disminución superior al 5\% para clasificar a las comunidades autónomas con un valor de un -2 , una disminución de entre un $5 \%$ y un $2 \%$ para clasificarlas con un -1 y una disminución menor al $2 \%$ para clasificarlas con un 0 .

- En el caso del cambio en la duración de la prestación y en las condiciones de acceso, la asignación de valores fue de tipo cualitativo, ya que las variables no fueron numéricas y se tuvo en cuenta el rango de opciones de cambio que se habían producido en el conjunto de las comunidades autónomas. En el caso de la valoración del cambio de las condiciones de acceso, la necesidad de sintetizar seis indicadores cualitativos ${ }^{1}$ obligó a establecer un criterio estándar para el conjunto de comunidades autónomas, de forma que:

- Se asignó un 0 cuando no se observó ningún cambio en las condiciones, solo se modificó una de ellas o hubo una que mejoró y otra que empeoró.

- Se asignó un -1 o un 1 cuando cambiaron dos condiciones en un mismo sentido, aunque hubiera una tercera que se modificara en el contrario.

- Se asignó un -1 o un 2 cuando cambiaron al menos tres condiciones en un mismo sentido, aunque hubiera alguna otra que se modificara en el contrario.

- En los casos de igual número de condiciones de mejora y empeoramiento, el equipo dirimió el empate, considerando la relevancia diferente de cada una de las condiciones afectadas.

Una vez asignados todos los valores, se calculó el valor promedio de cada dimensión, así como de las tres dimensiones conjuntamente para calcular el valor del cambio general. En función del resultado, se clasificó a las comunidades autónomas en tres grupos:

- Comunidades autónomas que tendieron a empeorar su comportamiento en RMI, con valores entre -2 y $-0,3$.

- Comunidades autónomas que mostraron cambios variables en su comportamiento en RMI, con valores entre $-0,2$ y 0,2 .

1. La complejidad de la valoración cualitativa del cambio en los indicadores puede implicar algún sesgo, si bien trabajos anteriores (Fernández Mállo, 2013) realizan un proceso de análisis similar. 
- Comunidades autónomas que tendieron a mejorar su comportamiento en RMI, con valores entre 0,3 y 2 .

El cruce de ambas clasificaciones (situación de la RMI al comienzo del periodo y cambio durante la crisis) dio lugar a la tipología final.

\section{Resultados}

La intensidad de las políticas de RMI en 2008 fue altamente heterogénea, tal y como se puede comprobar en la tabla 1 . Según los criterios utilizados para medir la intensidad de las RMI — el gasto por habitante, la tasa de cobertura en relación con la población en riesgo de pobreza y la cuantía básica de la prestación-, únicamente tres comunidades autónomas (Asturias, el País Vasco y Navarra) presentaron programas de RMI de intensidad reseñable. Entre ellas, Navarra y el País Vasco destacaron por mostrar los indicadores más elevados, puesto que obtuvieron la puntuación máxima (3) en todos los indicadores. Asturias obtuvo una puntuación media de 2,75 sobre 3. Es especialmente llamativa la distancia que existía en 2008 entre los resultados del País Vasco y el resto de comunidades autónomas en relación tanto con el gasto por habitante (91,5 euros, frente a la media de 10,7 euros), como con las tasas de cobertura sobre la población en riesgo de pobreza (un 76,9\% frente a un 9,8\%) y la cuantía básica de la prestación (616 euros frente a 403,4 euros).

En el lado opuesto, destacaron Murcia, Extremadura, Castilla-León, Islas Baleares y Galicia, con unas políticas de RMI débiles. El caso de Murcia era especialmente destacable, ya que su gasto por habitante en 2008 era inferior a un euro, su tasa de cobertura en relación con la población en riesgo de pobreza fue menor del $1 \%$ y la cuantía básica de su prestación (300 euros), la más baja del conjunto de comunidades autónomas. En una posición intermedia, se situó el resto de dichos entes territoriales, entre los que no se hallaron importantes diferencias respecto al comienzo del período.

El cambio producido a lo largo de la etapa de crisis en las diferentes comunidades autónomas ha variado según la dimensión considerada y ha estado probablemente condicionada por el punto de partida que tenían en 2008, según se ha descrito anteriormente.

La mayor parte de las comunidades autónomas ha mejorado en lo relativo a los recursos empleados y a la cobertura de la RMI entre 2008 y 2014 (tabla 1). En este sentido, son claramente subrayables, por ejemplo, los casos de la Comunidad Valenciana y de Aragón, que han multiplicado su gasto en RMI por habitante (considerando el cambio del IPC del periodo) por casi diez en estos siete años, tal y como se puede ver en la tabla 2. Extremadura y La Rioja también mostraron aumentos relevantes en su gasto en RMI durante el periodo. Por el contrario, el País Vasco fue el territorio que menos aumentó su gasto, si bien partía en 2008 de la posición más aventajada, con una gran distancia respecto al resto de comunidades autónomas. 
Tabla 1. Cambio en las tres dimensiones de las RMI de las comunidades autónomas entre 2008 y 2014, según la intensidad de la política al comienzo del periodo

\begin{tabular}{|c|c|c|c|c|}
\hline & & Empeora & Sin cambios & Mejora \\
\hline \multirow[t]{5}{*}{ Débil } & Islas Baleares & & & \\
\hline & Castilla y León & & & \\
\hline & Extremadura & & & \\
\hline & Galicia & & & \\
\hline & Murcia & & & \\
\hline \multirow[t]{9}{*}{ Media } & Andalucía & & & \\
\hline & Aragón & & & \\
\hline & Canarias & & & \\
\hline & Cantabria & & & \\
\hline & Castilla-La Mancha & & & \\
\hline & Madrid & & & \\
\hline & La Rioja & & & \\
\hline & Comunidad Valenciana & & - & \\
\hline & Cataluña & & & \\
\hline \multirow[t]{3}{*}{ Intensa } & Asturias & & & \\
\hline & Navarra & & & \\
\hline & País Vasco & & & \\
\hline
\end{tabular}

Recursos y cobertura.

Condiciones de acceso.

Características.

Fuente: elaboración propia.

En relación con las tasas de cobertura de población en riesgo de pobreza, estas aumentaron en casi todas las comunidades autónomas, especialmente en Castilla-La Mancha y Extremadura, con incrementos muy relevantes, si bien los puntos de partida eran realmente bajos. Así, por ejemplo, en Castilla-La Mancha, la población con riesgo de pobreza cubierta pasó de un $0,52 \%$ en 2008 a un 2,54\% en 2014 .

Las únicas comunidades autónomas que redujeron esta tasa fueron Andalucía, el País Vasco y Cataluña, con unas disminuciones del 52,5\%, del 19,4\% y del $9 \%$, respectivamente.

A diferencia de la dimensión de recursos y de cobertura, el comportamiento de las comunidades autónomas en relación con el cambio en las características de las RMI ha sido de mayor heterogeneidad. Se ha observado un mayor número de casos en los que estas han empeorado durante el periodo de crisis, especialmente entre aquellas comunidades autónomas que, en 2008, partían de una política de RMI débil (tablas 1 y 3). Así, en Castilla-La Mancha, la cuantía básica de la prestación se redujo en un 7,1\%, si bien su duración mejoró a partir de 2010, puesto que se eliminó el límite de 6 meses establecido hasta el momento. En Murcia, la cuantía también disminuyó durante los años de crisis en un 8,9\%, aunque no se produjo ninguna modificación en la duración de la misma. En Canarias, por su parte, 
Tabla 2. Indicadores sobre RMI según comunidades autónomas y año y porcentaje de cambio de cada uno de ellos en el periodo estudiado

\begin{tabular}{|c|c|c|c|c|c|c|c|c|}
\hline & & Andalucía & Aragón & Asturias & $\begin{array}{c}\text { Islas } \\
\text { Baleares }\end{array}$ & Canarias & Cantabria & $\begin{array}{l}\text { Castilla- } \\
\text { La Mancha }\end{array}$ \\
\hline \multirow{8}{*}{$\begin{array}{l}\text { Gasto en RMI } \\
\text { por habitante }\end{array}$} & 2008 & 4,76 & 1,80 & 21,30 & 3,38 & 2,78 & 6,19 & 0,72 \\
\hline & 2009 & 7,51 & 3,28 & 27,31 & 4,17 & 7,26 & 12,05 & 1,02 \\
\hline & 2010 & 7,65 & 7,37 & 29,99 & 4,90 & 8,35 & 11,99 & 1,15 \\
\hline & 2011 & 7,31 & 11,13 & 35,20 & 5,84 & 8,33 & 25,75 & 1,54 \\
\hline & 2012 & 8,21 & 14,63 & 41,72 & 5,29 & 9,30 & 25,68 & 1,42 \\
\hline & 2013 & 11,86 & 16,69 & 55,35 & 6,31 & 9,65 & 25,34 & 1,28 \\
\hline & 2014 & 10,79 & 21,62 & 77,57 & 8,40 & 12,59 & 29,51 & 2,66 \\
\hline & $14 / 08^{1}$ & $106,8 \%$ & $989,9 \%$ & $229,3 \%$ & $124,7 \%$ & $323,6 \%$ & $327,2 \%$ & $233,1 \%$ \\
\hline \multirow{7}{*}{$\begin{array}{l}\text { Tasa de cobertura (total) } \\
\text { sobre población con } \\
\text { riesgo de pobreza }\end{array}$} & 2009 & 5,57 & 4,84 & 16,17 & 2,98 & 1,31 & 5,63 & 0,52 \\
\hline & 2010 & 5,88 & 6,92 & 19,02 & 2,71 & 2,81 & 8,05 & 0,80 \\
\hline & 2011 & 6,03 & 7,41 & 16,67 & 3,43 & 2,47 & 9,76 & 2,11 \\
\hline & 2012 & 6,12 & 10,45 & 16,35 & 3,01 & 2,38 & 9,28 & 1,66 \\
\hline & 2013 & 7,04 & 12,67 & 18,69 & 3,43 & 2,41 & 12,23 & 1,53 \\
\hline & 2014 & 2,65 & 14,82 & 22,53 & 4,65 & 2,72 & 8,46 & 2,54 \\
\hline & $14 / 09$ & $-52,5 \%$ & $206,5 \%$ & $39,3 \%$ & $55,9 \%$ & $108,3 \%$ & $50,1 \%$ & $387,1 \%$ \\
\hline \multirow[t]{8}{*}{ Cuantía básica } & 2008 & 372,00 & 400,00 & 413,33 & 383,13 & 451,67 & 413,52 & 361,83 \\
\hline & 2009 & 386,68 & 424,00 & 432,09 & 392,38 & 467,49 & 421,79 & 369,07 \\
\hline & 2010 & 392,65 & 441,00 & 436,41 & 396,31 & 472,16 & 426,01 & 372,76 \\
\hline & 2011 & 397,67 & 441,00 & 442,96 & 405,52 & 472,16 & 426,01 & 372,76 \\
\hline & 2012 & 397,67 & 441,00 & 442,96 & 417,30 & 472,16 & 426,01 & 372,76 \\
\hline & 2013 & 400,09 & 441,00 & 442,96 & 425,70 & 472,16 & 426,01 & 372,76 \\
\hline & 2014 & 400,09 & 441,00 & 442,96 & 426,86 & 472,16 & 426,01 & 372,76 \\
\hline & $14 / 08$ & $-1,9 \%$ & $0,0 \%$ & $-3,1 \%$ & $0,7 \%$ & $-2,2 \%$ & $-7,7 \%$ & $-7,1 \%$ \\
\hline
\end{tabular}

1. Porcentaje del cambio corregido por el IPC 2008-2014.

Fuente: elaboración propia.

también se rebajó la cuantía de la prestación en un 2,2\%, aunque mejoró la duración de la misma en 2009, de 12 meses a 24, y, excepcionalmente, 6 meses más. Entre el resto de comunidades autónomas con intensidades mayores en su política de RMI al comienzo del periodo, destacaron los casos de la Comunidad Valenciana, Cantabria y Cataluña, con reducciones de la cuantía básica mayores al 5\% durante la crisis y rebajas de los periodos de duración en las dos últimas. En Cantabria, en 2012, se pasó de no establecer un tiempo máximo a limitarlo a 24 meses con posibles renovaciones, y en Cataluña, se produjeron restricciones de tiempo en varios años. En concreto, en 2011, la prestación pasó de prorrogarse anualmente a introducir una evaluación para poder optar a la misma. En 2013, además, se limitó, salvo algunas excepciones, la duración total de la prestación a 60 meses. Por último, en las comunidades autónomas con políticas que, en origen, eran de mayor intensidad, se observan mejoras en la duración de las prestaciones y 
Tabla 2. Indicadores sobre RMI según comunidades autónomas y año y porcentaje de cambio de cada uno de ellos en el periodo estudiado (continuación)

\begin{tabular}{rrrrrrrrrr}
\hline $\begin{array}{c}\text { Castilla } \\
\text { y León }\end{array}$ & Cataluña & Extremadura & Galicia & Madrid & Murcia & Navarra & País Vasco & La Rioja & $\begin{array}{c}\text { C. } \\
\text { Valenciana }\end{array}$ \\
\hline 4,43 & 10,38 & 1,55 & 6,84 & 6,60 & 0,59 & 16,76 & 91,53 & 2,17 & 0,48 \\
5,39 & 14,64 & 1,90 & 7,54 & 7,47 & 1,06 & 34,05 & 120,94 & 5,11 & 2,23 \\
6,42 & 21,27 & 2,01 & 8,06 & 9,13 & 1,93 & 48,40 & 143,83 & 8,84 & 3,23 \\
10,84 & 22,61 & 1,27 & 8,57 & 11,05 & 2,71 & 56,42 & 149,25 & 14,48 & 3,47 \\
13,13 & 17,17 & 1,50 & 9,62 & 13,02 & 2,69 & 47,01 & 156,59 & 18,26 & 2,86 \\
18,49 & 17,58 & 1,74 & 13,27 & 13,15 & 4,06 & 58,14 & 198,65 & 17,73 & 4,61 \\
22,05 & 21,55 & 15,95 & 16,03 & 15,35 & 4,89 & 76,47 & 198,89 & 21,47 & 5,33 \\
$353,3 \%$ & $85,2 \%$ & $838,9 \%$ & $111,9 \%$ & $111,0 \%$ & $654,8 \%$ & $317,1 \%$ & $95,9 \%$ & $797,8 \%$ & $909,5 \%$ \\
\hline 1,79 & 6,96 & 1,79 & 2,96 & 3,63 & 0,65 & 30,57 & 76,89 & 3,62 & 1,58 \\
1,56 & 9,66 & 1,72 & 3,68 & 5,54 & 0,84 & 32,92 & 59,75 & 5,83 & 1,54 \\
3,34 & 5,36 & 1,09 & 3,97 & 6,41 & 1,28 & 46,89 & 68,55 & 9,81 & 1,86 \\
3,75 & 4,56 & 1,06 & 4,41 & 6,64 & 1,52 & 40,08 & 58,83 & 11,79 & 2,75 \\
4,59 & 5,64 & 1,01 & 6,23 & 7,22 & 1,90 & 55,35 & 74,79 & 10,97 & 2,94 \\
6,79 & 6,33 & 7,54 & 7,09 & 8,87 & 1,92 & 54,69 & 61,96 & 11,93 & 3,30 \\
$279,3 \%$ & $-9,0 \%$ & $320,9 \%$ & $139,8 \%$ & $144,5 \%$ & $195,4 \%$ & $78,9 \%$ & $-19,4 \%$ & $230,0 \%$ & $108,6 \%$ \\
\hline 387,68 & 400,38 & 387,68 & 387,68 & 354,00 & 300,00 & 510,00 & 616,00 & 345,13 & 373,89 \\
395,43 & 410,02 & 387,68 & 395,43 & 370,00 & 300,00 & 561,60 & 640,64 & 352,70 & 381,37 \\
399,38 & 414,12 & 399,38 & 399,38 & 375,55 & 300,00 & 633,30 & 650,19 & 186,38 & 385,18 \\
426,00 & 423,70 & 399,38 & 399,38 & 375,55 & 300,00 & 641,40 & 658,50 & 360,18 & 385,18 \\
426,00 & 423,70 & 399,38 & 399,38 & 375,55 & 300,00 & 641,40 & 612,41 & 365,23 & 385,18 \\
426,00 & 423,70 & 399,38 & 399,38 & 375,55 & 300,00 & 548,51 & 662,51 & 368,83 & 385,18 \\
426,00 & 423,70 & 426,00 & 399,38 & 375,55 & 300,00 & 548,51 & 665,90 & No indica & 385,18 \\
$0,1 \%$ & $-5,6 \%$ & $0,3 \%$ & $-6,9 \%$ & $-3,7 \%$ & $-8,9 \%$ & $-1,7 \%$ & $-2,5 \%$ & $-3,0 \%$ & $-6,3 \%$ \\
\hline
\end{tabular}

reducciones de las cuantías básicas comparativamente más bajas en relación con el conjunto de comunidades autónomas.

Otro de los indicadores en esta segunda dimensión que revierte gran interés para entender el comportamiento diferencial de las comunidades autónomas a lo largo de la crisis es el relativo a garantizar, en base a su declaración como derecho subjetivo o equivalente, la financiación de la prestación. En este sentido, nueve comunidades autónomas garantizaron la financiación de la RMI a lo largo del periodo, pero otras cuatro - Islas Baleares, Canarias, Castilla-La Mancha y Comunidad Valenciana - dejaron de garantizarla a lo largo de la crisis. Resulta llamativo el caso de esta última, en la que, a pesar de que se reconoce la renta garantizada de ciudadanía como un derecho subjetivo, en la Ley de presupuestos de 2010 no se reconoció ya como crédito ampliable en caso necesario (Resolución de 5 de marzo de 2010). En positivo, destacaron los casos de Castilla-León, Extremadura, Galicia y Murcia, que pasaron a garantizar sus RMI a partir de los años 2010 o 2011. 
Tabla 3. Cambio en las condiciones de acceso a las RMI en las diferentes comunidades autónomas durante el periodo 2008-2014

\begin{tabular}{|c|c|c|c|c|c|c|}
\hline & $\begin{array}{c}\text { Persona } \\
\text { perceptora } \\
\text { (persona o UC) }\end{array}$ & $\begin{array}{c}\text { Edad } \\
\text { mínima }\end{array}$ & $\begin{array}{c}\text { Prueba } \\
\text { de rentas }\end{array}$ & $\begin{array}{c}\text { Tiempo de } \\
\text { cohabitación } \\
\text { en la unidad } \\
\text { familiar o } \\
\text { convivencia }\end{array}$ & $\begin{array}{c}\text { Tiempo de } \\
\text { empadronamiento }\end{array}$ & $\begin{array}{l}\text { Tiempo de } \\
\text { residencia }\end{array}$ \\
\hline Andalucía & $=$ & $=$ & $\uparrow 2010$ & $=$ & $=$ & $=$ \\
\hline Aragón & $=$ & $\uparrow 2012$ & $\downarrow 2010$ & $=$ & $=$ & $=$ \\
\hline Asturias & $=$ & $\uparrow 2014$ & $\downarrow 2011$ & $=$ & $\uparrow 2009$ & $=$ \\
\hline Islas Baleares & $=$ & $\uparrow 2012$ & $=$ & $=$ & $=$ & $=$ \\
\hline Canarias & $\uparrow 2012$ & $=$ & $=$ & $=$ & $=$ & $=$ \\
\hline Cantabria & $=$ & $\uparrow 2012$ & $\downarrow 2010$ & $=$ & $=$ & $=$ \\
\hline Castilla-La Mancha & $=$ & $\uparrow 2013$ & $=$ & $\downarrow 2011$ & $=$ & $=$ \\
\hline Castilla y León & $\uparrow 2010$ & $=$ & $=$ & $=$ & $\uparrow 2013$ & $\uparrow 2013$ \\
\hline Cataluña & $=$ & $\begin{array}{l}\downarrow 2011 \\
\uparrow 2014^{1}\end{array}$ & $=$ & $=$ & $=$ & $\downarrow 2011$ \\
\hline Extremadura & $\downarrow 2011$ & $\begin{array}{l}\uparrow 2012 \\
\downarrow 2013\end{array}$ & $=$ & $\uparrow 2012$ & $=$ & $=$ \\
\hline Galicia & $=$ & $=$ & $\begin{array}{l}\downarrow 2011 \\
\uparrow 2014^{3}\end{array}$ & $=$ & $\uparrow 2014$ & $\uparrow 2014$ \\
\hline Madrid & $=$ & $\downarrow 2014$ & $=$ & $=$ & $\downarrow 2014$ & $=$ \\
\hline Murcia & $=$ & $\uparrow 2013$ & $=$ & $=$ & $=$ & $\downarrow 2013$ \\
\hline Navarra & $=$ & $=$ & $=$ & $=$ & $=$ & $\downarrow 2012$ \\
\hline País Vasco & $=$ & $=$ & $\uparrow 2014$ & $=$ & $=$ & $\downarrow 2013$ \\
\hline La Rioja & $\downarrow 2011$ & $\begin{array}{l}\uparrow 2011 \\
\downarrow 2014^{2}\end{array}$ & $\begin{array}{l}\downarrow 2010 \\
\uparrow 2014^{4}\end{array}$ & $=$ & $\begin{array}{l}\downarrow 2012 \\
\uparrow 2014^{5}\end{array}$ & $\begin{array}{l}\downarrow 2012 \\
\uparrow 2014^{5}\end{array}$ \\
\hline C. Valenciana & $=$ & $=$ & $=$ & $=$ & $=$ & $\uparrow 2010$ \\
\hline
\end{tabular}

1. En 2011, se amplió la edad necesaria a 25 años y disminuyó de nuevo en 2014.

2. En 2011, se ampliaron los supuestos para que pudieran percibirla los menores de 25 años, pero en 2014 empeoró, porque se estableció la edad máxima en 65 años.

3. Empeoró en 2010 (se fijó la cuantía en un 70\% IPREM y se congeló), pero mejoró en 2014 (se quitó la cantidad de referencia).

4. Empeoró en 2010 (se fijó la cuantía en un 70\% IPREM y se congeló), pero mejoró en 2014 (se ampliaron las cuantías tope).

5. En 2012, aumentó el periodo de empadronamiento y de residencia de 12 a 36 meses, pero se volvieron a reducir en 2014.

Fuente: elaboración propia.

Una lectura sobre el cambio en las condiciones de acceso por comunidades autónomas (tabla 3) advierte que, en la mayor parte de las mismas, no se han producido variaciones sustanciales, si bien un análisis más en detalle ofrece claves interesantes. En este sentido, parece que la variación mayor, que en lo fundamental ha sido hacia su empeoramiento, se ha producido en el tiempo de residencia exigido para poder acceder a la prestación. A partir de 2011, este requisito se ha endurecido en Cataluña, Murcia, País Vasco, Navarra y La Rioja. Por el contrario, la condición que parece haber mejorado en mayor medida ha sido la edad mínima exigida para percibir la prestación, que 
mejoró a lo largo de la crisis en seis comunidades autónomas y varió tanto en positivo como en negativo en otras tres. En la mayor parte de los casos, se rebajó el límite de 25 años a 18, e incluso, en determinadas circunstancias, como la tenencia de menores a cargo, se pasó a considerar edades menores. Por su parte, la condición que menos modificaciones ha sufrido ha sido la del tiempo de cohabitación exigido a la unidad familiar o de convivencia, que tan solo empeoró en 2011 en Castilla-La Mancha y mejoró en Extremadura en 2012. Algunas comunidades autónomas, como Castilla y León, destacan por la mejora observada en la mitad de las condiciones (pasó de consideración de unidad de convivencia a persona individual como perceptora de la prestación, exigencia únicamente realizada a la persona titular del tiempo de empadronamiento y del tiempo de residencia establecidos), y La Rioja, por el agravamiento de gran parte de ellas. Asimismo, Madrid mostró un claro empeoramiento de sus condiciones de acceso, con un endurecimiento, tanto de la edad mínima de acceso como del tiempo de empadronamiento exigido. Las comunidades autónomas que con más frecuencia endurecieron sus condiciones de acceso a la prestación partían de una intensidad media en sus RMI a comienzo del periodo.

\section{Tipología de comunidades autónomas según su comportamiento} en RMI en función de su situación de partida y su cambio en época de crisis

Una vez realizado el análisis sobre la evolución de cada una de las tres dimensiones consideradas, se describe a continuación la tipología de comunidades autónomas construida (tabla 4). En términos generales, se observó que las RMI mejoraron a lo largo del periodo de crisis en la gran mayoría de comunidades autónomas, independientemente de su punto inicial en 2008.

Entre aquellas que se clasificaron como débiles al inicio del periodo, se observó una mejoría en todas ellas durante la etapa de crisis. Entre estas, destacó Castilla-León, que mejoró claramente su RMI, sobre todo en relación con los indicadores de recursos y cobertura, así como con las condiciones de acceso.

Asimismo, las comunidades autónomas con RMI de mayor intensidad al comienzo del periodo también mejoraron sus políticas de rentas, incluso aquellas, como el País Vasco, que partían de los indicadores más favorables. En este caso, su política mejoró en dos de las tres dimensiones consideradas. Asturias, por su parte, fue la comunidad autónoma con una situación de partida definida como intensa que mejoró más claramente durante el periodo de crisis. Entre las comunidades autónomas con RMI de intensidad media al comienzo del periodo, hubo mayor heterogeneidad. Si bien la mayoría de ellas mostró un comportamiento ligeramente positivo en sus RMI, Cataluña y La Rioja no ofrecieron comportamientos positivos, ya que la primera vio empeorada su política de RMI en términos generales durante el periodo de crisis, mientras que La Rioja mostró un comportamiento variable, de cierta mejora en algunas dimensiones, pero de gran deterioro en las condiciones de acceso y empeoramiento en algunas de sus dimensiones. 
Tabla 4. Clasificación de comunidades autónomas según la situación de partida de las RMI y cambio en el periodo 2008-2014

\begin{tabular}{llccc}
\hline & & \multicolumn{2}{c}{ Cambio } \\
\cline { 3 - 5 } & & $\begin{array}{c}\text { Tendencia } \\
\text { a empeorar }\end{array}$ & $\begin{array}{c}\text { Con cambios } \\
\text { variables }^{1}\end{array}$ & $\begin{array}{c}\text { Tendencia } \\
\text { a mejorar }\end{array}$ \\
\hline $\begin{array}{l}\text { Situación de } \\
\text { partida de la } \\
\text { política }\end{array}$ & Débil & & & $\begin{array}{c}\text { Islas Baleares, Castilla y León, } \\
\text { Extremadura, Galicia y Murcia. }\end{array}$ \\
\cline { 2 - 5 } & Media & Cataluña. & La Rioja. & $\begin{array}{c}\text { Andalucía, Aragón, Canarias, } \\
\text { Cantabria, Castilla-La Mancha, } \\
\text { Madrid y Comunidad Valenciana. }\end{array}$ \\
\cline { 2 - 5 } & Intensa & & Asturias, Navarra y el País Vasco. \\
\hline
\end{tabular}

1. Esta categoría recoge comunidades autónomas que mostraron cambios variables, tanto de mejora como de empeoramiento, no clasificables en las otras categorías.

Fuente: elaboración propia.

\section{Conclusiones y discusión}

El análisis realizado permite confirmar, con respecto a la evolución de las RMI durante el periodo 2008-2014 en el conjunto del Estado español, la existencia de una importante variabilidad y heterogeneidad de estos programas ya identificada en otros estudios (Fernández Maíllo, 2013). Asimismo, los resultados muestran que, en el contexto de crisis, la respuesta de las comunidades autónomas no ha sido homogénea en la gestión de las RMI. En este sentido, entre los elementos que definen la mayor o menor intensidad de la política aplicada en la regulación y en la aplicación de las rentas mínimas, cabe señalar que el periodo de crisis se ha caracterizado por una cierta mejoría general en el comportamiento de las comunidades autónomas en sus RMI, independientemente de su situación de partida. Ello se ha debido, muy probablemente, a dos factores: por una parte, a una situación de partida en el que las RMI estaban, en términos generales, poco desarrolladas $\mathrm{y}$, por otra, a los requerimientos inmediatos que ha exigido un escenario de creciente vulnerabilidad social en época de crisis y que ha obligado a las comunidades autónomas a tomar medidas paliativas para mejorar, en alguna medida, sus políticas de mantenimiento de rentas.

Sin embargo, esta cierta mejoría no permite obviar el hecho de que persiste una cierta desigualdad en el acceso a las RMI y una insuficiencia respecto a las realidades sociales que acompaña el contexto sometido a análisis. Así, ante un escenario de una cada vez mayor desigualdad en España (Intermón-Oxfam, 2016), esta panorámica de las RMI apunta, como ya lo hacían otros trabajos (Fernández Maíllo, 2013; Arriba González de Durana, 2014), hacia la persistencia de cierta falta de equidad territorial en el sistema de protección, así como hacia una cobertura todavía insuficiente de las necesidades de los colectivos más vulnerables. En este sentido, a excepción del País Vasco y Navarra, donde más de la mitad de la población en riesgo de pobreza era perceptora de una RMI en 2014, la gran mayoría de comunidades autónomas cubrían a menos del $10 \%$ de la población en riesgo. 
Asimismo, el análisis realizado nos permite destacar algunas cuestiones relativas a las tres dimensiones identificadas para este trabajo. En primer lugar, respecto a las condiciones de acceso a la RMI, según los resultados obtenidos en nuestro estudio, resulta relevante el hecho de que cinco comunidades autónomas hayan endurecido los requisitos respecto al tiempo de residencia exigido para poder optar a la prestación: el País Vasco, Navarra, Murcia, Cataluña y La Rioja ${ }^{2}$. Madrid, si bien no incrementa el tiempo de residencia exigido para optar a la prestación, sí endurece el tiempo exigido de empadronamiento. Esta cuestión parecería indicar, como ya señalan estudios anteriores (Moreno et al., 2015), que los discursos que asocian inmigración con cobro indebido de prestaciones estarían ejerciendo cierto impacto en la definición de unas RMI especialmente exigentes para colectivos como el inmigrante, que acusan ya una importante vulnerabilidad. Por otro lado, en su conjunto, el resto de condiciones de acceso a las RMI no han experimentado cambios relevantes a lo largo de la crisis, si bien cabría destacar la mejora en la condición relativa a la edad media exigida para percibir la prestación, que ha sido rebajada de 25 a 18 años en varias comunidades autónomas.

En segundo lugar, respecto a la dimensión de las características básicas de la RMI, creemos prioritario llamar la atención sobre los resultados de nuestro estudio en relación con la cuantía de las prestaciones. El análisis de la cuantía y de la duración de la prestación, así como de su garantía de financiación, nos permite observar, entre otras cuestiones, cómo empeora la situación entre aquellas comunidades autónomas que, en 2008, partían ya de una política de RMI débil. Además, también algunas comunidades autónomas que partían de una RMI de mayor intensidad muestran, por ejemplo, reducciones de la cuantía básica mayores que el 5\% durante la crisis. Es el caso de la Comunidad Valenciana, Cantabria y Cataluña. En este contexto, cabe destacar el hecho de que España tiene una de las tasas más altas de pobreza en los hogares con niños y niñas de Europa (Cantó y Ayala, 2014), lo que afecta de manera especial a las familias de nacionalidad no española. La congelación de las cuantías de las rentas mínimas de inserción en la mayoría de las comunidades desde el año 2010 muestra una falta de adecuación de las mismas respecto a la cobertura de las necesidades básicas. Sería oportuno que se abrieran líneas de investigación futuras que pudieran indagar en esta problemática.

Por último, los resultados obtenidos en el análisis de la dimensión de los recursos y de la cobertura de la RMI permiten concluir que la mayor parte de las comunidades autónomas ha mejorado entre 2008 y 2014. En dicho aspecto, destacan los casos de la Comunidad Valenciana y Aragón, que multiplican su gasto en RMI por habitante. Por el contrario, el País Vasco fue la comunidad con menor incremento en el gasto, si bien partía de una mejor posición respecto al resto de comunidades. Las tasas de cobertura de población en riesgo de pobreza también mejoran en casi todas las comunidades autónomas, si bien es importante señalar que, en 2008, estas tasas eran realmente bajas.

2. Desde el año 2014, el tiempo de residencia necesario vuelve a ser de 12 meses. 
La lectura de los resultados expuestos nos permite discutir sobre el desarrollo reciente de las RMI en el conjunto del Estado español. Así, parecería reforzarse la idea de unos dispositivos de rentas mínimas fuertemente asentados en la tradición asistencialista del estado de bienestar propia de los países del sur de Europa, y lejos todavía del modelo de protección social centrado en el derecho a la ciudadanía que protagonizan los países nórdicos (Fernández, 2015). El estudio que hemos presentado trata precisamente de ofrecer algunos datos que acompañen la discusión, no solo sobre la realidad de las RMI en el conjunto del Estado español, sino también sobre la relevancia de la definición de estas rentas para la obtención de una mejor o peor respuesta a las necesidades sociales en un determinado contexto.

Así, el trabajo muestra las diferencias territoriales en intensidad de las RMI en el inicio del periodo de análisis, siendo en su gran mayoría políticas de intensidad media o débil. El País Vasco, Navarra y Asturias constituyen aquí los únicos casos de comunidades con una RMI de intensidad alta. El análisis posterior respecto a la evolución de las RMI en el periodo de crisis nos permite concluir que, si bien se aprecia una mejora general de las políticas de RMI en el conjunto de las comunidades autónomas, tan solo dos lo hacen de manera sustantiva (Castilla y León y Asturias) y el resto presenta alguna dimensión en la que ha empeorado su situación de partida (de manera especial, las relativas a la duración, a la cuantía y a la garantía de financiación).

Debemos señalar de nuevo la importancia de atender a la evolución de estas RMI en relación con el contexto en el que se implementan. Así, realidades tales como la aparición de nuevos riesgos sociales (Rodriguez Cabrero 2014) y el constante incremento de demanda de estas prestaciones, fruto del aumento del desempleo, el ascenso de la pobreza (la tasa de pobreza severa se ha duplicado desde 2007 hasta 2014) y la ampliación de la privación material de los hogares y del crecimiento de la exclusión social (Arriba González de Durana, 2014), apuntan a la necesidad de profundizar en trabajos que midan la adecuación de las mismas a sus objetivos primarios de lucha contra la pobreza y contra la exclusión social. En este sentido, también sería interesante abrir líneas de investigación sobre cuestiones tales como la propia gestión de las RMI (mediante entrevistas al personal encargado de su tramitación) o el análisis de la relación entre variables tales como el grado de cobertura y las condiciones de acceso, de cara a ofrecer una lectura más completa sobre la realidad de las RMI.

\section{Referencias bibliográficas}

Aguilar, M.; Gaviria, M.; Laparra, M. (1995). La caña y el pez: El salario social en las comunidades autónomas, 1989-1994. Madrid: Fundación Foessa.

Arriba González de Durana, A. (2014). «El papel de la garantía de mínimos frente a la crisis». VII Informe sobre exclusión y desarrollo social en España 2014 [en línea]. Madrid: Fundación Foessa. Documento de trabajo 5.7. <http://www.foessa2014. es/informe/uploaded/documentos_trabajo/04112014003757_2107.pdf>. 
Arriba González de Durana, A. y Guinea, D. (2008). «Protección social, pobreza y exclusión social: El papel de los mecanismos de protección de rentas». VI Informe sobre exclusión y desarrollo social en España. Madrid: Fundación Foessa, 325-346.

Ayala, L. (2012). "El desbordamiento del sistema de garantía de ingresos en la crisis». Gaceta Sindical [en línea], 19. 330-358. <https://dialnet.unirioja.es/servlet/ articulo? codigo $=4091520>$.

Bacigalupe et al. (2015a). "Why is there so much controversy regarding the population health impact of the great recession?: Reflections on three case studies». International Journal of Health Services [en línea]. <http://dx.doi.org/10.1177/0020731415611634>.

- (2015b). «Austeridad y privatización sanitaria en época de crisis: ¿Existen diferencias entre las comunidades autónomas?». Gaceta Sanitaria [en línea], 30 (1), 47-51. <http://dx.doi.org/10.1016/j.gaceta.2015.10.003>.

Bieling, H.J. (2012). "EU facing the crisis: Social and employment policies in times of tight budgets». Transfer: European Review of Labour and Research [en línea], 18 (3), 255-271. <http://dx.doi.org/10.1177/1024258912448591>.

Cantó Sánchez, O. y Ayala Cañón, L. (2014). Políticas públicas para reducir la pobreza infantil en España: Análisis de impacto [en línea]. Madrid: UNICEF Comité Español. <http://solidaria.unicef.es/pdf/UCE_politicas_para_reducir_pobr_ infantil_espana_14.pdf>.

CES (2016). Memoria sobre la situación socioeconómica y laboral de España 2015 [en línea]. Madrid: Consejo Económico y Social de España. <http://www.ces.es/documents/10180/3933980/Memoria_Socioeconomica_CES2015.pdf>.

Comisión Europea (2014). Taking Stock of the Europe 2020 Strategy for Smart, Sustainable and Inclusive Growth [en línea]. Bruselas. <http://ec.europa.eu/europe2020/ pdf/europe2020stocktaking_en.pdf>.

DeEming, C. (2015). «Defining Minimum Income (and Living) Standards in Europe: Methodological Issues and Policy Debates». Social Policy and Society [en línea], 16 (1). $<$ http://dx.doi.org/10.1017/s147474641500041x>.

De La Cal, M.L y De La Fuente, J. M (2010). «Algunos interrogantes en torno a los programas de inclusión activa como estrategia de lucha contra la pobreza y la exclusión social». Lan Harremanak, 22, 45-89.

Díaz-Chao, A.; Ficapal-Cusí, P. y Torrent-Sellens, J. (2015). «Determinantes multidimensionales en la calidad percibida del empleo: Evidencia empírica para España». Revista Internacional de Sociología [en línea], 73 (1). <http://dx.doi.org/10.3989/ris.2013.03.25>.

Díaz-Pulido, J.M.; Pino, E. del y Palop, P. (2012). «Los determinantes de la satisfacción con las políticas de bienestar del Estado autonómico». Revista Internacional de Sociología [en línea], 139, 45-84. <http://dx.doi.org/10.5477/cis/reis.139.45>.

EAPN (2010). Working document on a Framework on a Directive on Minimum Income [en línea]. Bruselas. European Anti-poverty Network. https://www.eapn.eu/ images/stories/docs/EAPN-position-papers-and-reports/Working-Paper-on-aFramework-Directive-EN-FINAL.pdf.

Fernández, G. (coord.) (2015). Hacia un sistema inclusivo de garantía de rentas en España: Diferentes alternativas de desarrollo [en línea]. Madrid: Fundación Foessa. $<$ http://www.foessa.es/publicaciones_compra.aspx?Id=5201\&Diocesis=42\&Idi oma $=1>$. 
Fernández García, T. (2012). «El estado de bienestar frente a la crisis política, económica y social». Portularia [en línea], XII, 3-12. <http://dx.doi.org/10.5218/prts.2012.0001>.

Fernández García, T. y Andrés Cabello, S. (2015). «Crisis y estado de bienestar: Las políticas sociales en la encrucijada». Tendencias y Retos [en línea], 20 (1), 119132. <http://revistas.lasalle.edu.co/index.php/te/article/view/3463>.

Fernández Maíllo, G. (2013). "El aumento de la fragilidad de las Rentas Mínimas de Inserción durante la crisis». Documentación Social [en línea], 166, 169-191. $<$ https://dialnet.unirioja.es/servlet/articulo?codigo=4316886>.

Figari, F.; Matsaganis, M. y Sutherland, H. (2013). «Are European social safety nets tight enough?: Coverage and adecuacy of Minimun Income schemes in $14 \mathrm{EU}$ countries». International Journal of Social Wellfare [en línea], 22, 3-14. <http://dx.doi.org/10.1111/j.1468-2397.2012.00885.x>.

Flores Paredes, J. y Nieto Solís, J.A. (2013). «Políticas sociales ante la crisis: Una comparación entre la UE y Latinoamérica». Revista de Economía Mundial [en línea], 36, 211-237. <http://www.redalyc.org/pdf/866/86632963008.pdf>.

Fuenmayor Fernández, A. y Granell Pérez, R. (2013). «Las Rentas Mínimas de Inserción autonómicas: Simulación de sus efectos sobre la pobreza». XX Encuentro de Economía Pública [en línea]. Sevilla, 1 de febrero. <https://dialnet.unirioja.es/ servlet/articulo? codigo $=4806417>$.

Gordillo Pérez, L. (2013). «La garantía de los derechos sociales en un contexto de crisis y políticas de austeridad». Nuevas Políticas Públicas: Anuario multidisciplinar para la modernización de las Administraciones Públicas [en línea], 8, 77-90. <https:// dialnet.unirioja.es/servlet/articulo?codigo $=5186032>$.

Gutiérrez, E. (2014). «Nueva pobreza y Renta Mínima de Inserción». Dossier Catalunya Social [en línea]. Barcelona: Tercer Sector Social. <http://www.tercersector. cat/sites/www.tercersector.cat/files/dossier_nueva_pobreza_y_renda_minima_de_ insercion_mayo_2014_castellano.pdf>.

Instituto Nacional de Estadística (INE) (2015). «Tasas de paro por sexo y grupo de edad». Datos básicos [en línea]. Madrid: Instituto Nacional de Estadística. $<$ http://www.ine.es/>.

InTERmón-OXfam (2016). Una economía al servicio del 1\% [en línea]. Informe de Intermón-Oxfam 210. <https://www.oxfam.org/sites/www.oxfam.org/files/file_ attachments/bp210-economy-one-percent-tax-havens-180116-es_0.pdf>.

Laparra, M. (2013). «Riesgos de pobreza, ingresos mínimos y servicios sociales». Cuadernos del Círculo Cívico de Opinión [en línea], 10, 13-39. http://www.circulocivicodeopinion.es/download/pdf/CUADERNOS\%2010\%20def1.pdf.

Laparra, M. y Ayala, L. (2009). El sistema de garantía de ingresos minimos en España y la respuesta urgente que requiere la crisis social [en línea]. Madrid: Cáritas y Fundación Foessa. <http://www.caritas-zaragoza.org/biblio/ficheros/0418388001247157176.pdf>.

Lavia Martínez, C.; Moreno Márquez, G. y Aierdi Urraza, X. (2014). «Diferencias en el uso de los programas de Rentas Mínimas según nacionalidad: La Renta de Garantía de Ingresos en la Comunidad Autónoma Vasca». Revista Internacional de Sociología [en línea], 72 (3), 633-660. <http://dx.doi.org/10.3989/ris.2013.01.23>.

Lorenzo Gilsanz, F. (comp.) (2014). VII Informe sobre exclusión y desarrollo social en España 2014 [en línea]. Madrid: Fundación Foessa. <http://www.foessa2014.es/ informe/uploaded/descargas/VII_INFORME.pdf>. 
Márquez Covarrubias, H. (2012). «Crisis del sistema capitalista mundial: Paradojas y respuestas». Polis [en línea], 27. <http://dx.doi.org/10.4067/s0718-65682010000300020>.

Méndez, R.; Abad, L. y Echaves, C. (2015). Atlas de la crisis: Impactos socioeconómicos y territorios vulnerables en España. Valencia: Tirant Humanidades.

Moreno Márquez, G.; Fouassier Zamalloa, M. y Martín Herrero, M.J. (2015). «Política de garantía de ingresos y población de origen extranjero en el País Vasco: Evidencias empíricas y deslegitimación discursiva». Zerbitzuan [en línea], 68, 51-60. <http://dx.doi.org/10.5569/1134-7147.58.05>.

Paleo, N. y Alonso, A. (2014). «¿Es solo una cuestión de austeridad?: Crisis económica y políticas de género en España». Investigaciones Feministas [en línea], 5, 36-68. <http://dx.doi.org/10.5209/rev_infe.2014.v5.47987>.

Parlamento Europeo (2009). Active Inclusion of People Excluded from the Labour Market [en línea]. Bruselas: Parlamento Europeo. <https://publications.europa. eu/en/publication-detail/-/publication/b8272e05-51d5-40e1-9796-e69224052f6f/ language-en>.

Pérez Orozco, A. (2012). "Crisis multidimensional y sostenibilidad de la vida». Investigaciones Feministas [en línea], 1, 29-53. <http://dx.doi.org/10.5209/rev_infe.2011.v2.38603>.

Pontusson, J. y Raess, D. (2002). "How (and why) is this time different?: The politics of economic crisis in western Europe and the United States». Annual Review of Political Science [en línea], 15, 13-33. <http://dx.doi.org/10.1146/annurev-polisci-031710-100955>.

Recio Andreu, A. (2013). «Esta crisis como problema sistémico». Vinculos de Historia, 2, 83-96.

«Resolución de 5 de marzo de 2010, de la consellera de Bienestar Social, por la que se da publicidad a los créditos que financian las ayudas convocadas mediante la Orden de 31 de julio de 2008 por la que se regulan las bases de convocatoria de la prestación Renta Garantizada de Ciudadanía, para el ejercicio 2010». Diari Oficial de la Comunitat Valenciana, 6244, 13988 (13 abril 2010).

Rodríguez Cabrero, G. (coord.) (2014). Estado de bienestar en España: Transformaciones y tendencias de cambio en el marco de la Unión Europea [en línea]. Madrid: Fundación Foessa. VII Informe sobre Exclusión y Desarrollo Social en España. <http://www.foessa2014.es/informe/uploaded/capitulos/pdf/05_Capitulo_5.pdf>.

RolNick, R. (2013). «Late neoliberalism: The financialisation of homeownership and housing rights». International Journal of Urban and Urban and Regional Research [en línea], 37 (3), 1058-1066. <http://dx.doi.org/10.1111/1468-2427.12062>.

SAnzo González, L. (2013). «La política de garantía de ingresos en Euskadi». Zerbitzuan [en línea], 53, 9-18. <http://dx.doi.org/10.5569/1134-7147.53.01>.

Shahidi, F.V. (2015). "Welfare Capitalism in Crisis: A Qualitative Comparative Analysis of Labour Market Responses to the Great Recession». Journal of Social Policy [en línea], 1 (4), 1-28. <http://dx.doi.org/10.1017/s004727941500029x>.

Subirats, J. (2012). «Nuevos tiempos, ¿nuevas políticas públicas?: Explorando caminos de respuesta». Revista del CLAD Reforma y Democracia [en línea], 54, 5-32. <http://www.redalyc.org/articulo.oa?id=357533686001>. 
Zalakain, J. (2014). «El papel de los sistemas de garantía de ingresos en el abordaje de la pobreza en el empleo: La experiencia del País Vasco». Lan Harremanak [en línea], 31 (II), 36-62. <http://www.ehu.eus/ojs/index.php/Lan_Harremanak/article/view/15167>. 